\title{
"Life histories" is a more appropriate term than "ecotypes" to describe ocean- and stream-maturing Pacific lamprey Entosphenus tridentatus
}

\author{
Benjamin Clemens ${ }^{1,2}$ and Carl Schreck ${ }^{3}$ \\ ${ }^{1}$ Oregon State University \\ ${ }^{2}$ Oregon Department of Fish and Wildlife \\ ${ }^{3}$ US Geological Survey, Oregon Cooperative Fish and Wildlife Research Unit, USGS
}

August 28, 2020

\begin{abstract}
The scientific literature includes a plethora of terms to denote within-species phenotypic diversity (e.g., morphotypes; ecotypes; ecomorphotypes; ecophenotypes; polymorphisms; and life histories). Here we discuss a particular situation in which different terms (ecotypes and life histories) have been used to describe the same within-species diversity of adult Pacific lamprey Entosphenus tridentatus, note an important challenge in using "ecotypes", and conclude with a recommendation to use "life histories" to describe the ocean- and stream-maturing forms of Pacific lamprey.
\end{abstract}

\begin{abstract}
The scientific literature includes a plethora of terms to denote within-species phenotypic diversity (e.g., morphotypes; ecotypes; ecomorphotypes; ecophenotypes; polymorphisms; and life histories). Here we discuss a particular situation in which different terms (ecotypesand life histories) have been used to describe the same within-species diversity of adult Pacific lamprey Entosphenus tridentatus, note an important challenge in using "ecotypes", and conclude with a recommendation to use "life histories" to describe the ocean- and stream-maturing forms of Pacific lamprey.

The scientific literature includes a plethora of terms to denote within-species phenotypic diversity. These include morphotypes (Lessios and Weinberg 1994; Chavarie et al. 2013; Collyer et al. 2015); ecotypes (Gregor 1944; Arostegui et al. 2018; Cruz-Font 2019); ecomorphotypes (Baker et al. 1998; Segura-Trujillo et al. 2018; Kloh et al. 2019); ecophenotypes (Schönborn and Peschke 1988; Sorensen and Lindberg 1991; Proćków et al. 2018); polymorphisms (Skúlason and Smith 1995; Skúlason et al. 2019; Jamie and Meier 2020); and life histories (Stearns 1989; Winemiller and Rose 1992). Several of these terms have common roots, and are often used inter-changeably or in combination (e.g., Baker et al. 1998; Chavarie et al. 2013; Brannon et al. 2004; Wood et al. 2008; Palacios et al. 2012). The use of these terms may suggest perceived or actual novelty, a unique take on biological phenomena, or an attempt to follow precedents. Here we discuss a particular situation in which different terms (ecotypes and life histories) have been used to describe the same within-species diversity of adult Pacific lampreyEntosphenus tridentatus, note an important challenge in using "ecotypes", and conclude with a recommendation to use "life histories" to describe the ocean- and stream-maturing forms of Pacific lamprey.

The Pacific lamprey is a jawless and boneless anadromous fish from a primitive taxon (Petromyzontiformes; Docker et al. 2015; Potter et al. 2015). Parker et al. (2019) genotyped Pacific lamprey at adaptive and neutral single nucleotide polymorphisms and measured the proportional egg mass of females to identify
\end{abstract}


a genetic basis for phenotypic variation in ocean- and stream-maturing lamprey. Their research offers an important contribution to the understanding of evolution and ecology in general, and of Pacific lamprey in particular. The results of Parker et al. support the conclusions of Clemens et al. (2013), who initially identified this same diversity from comprehensive phenotypic characteristics that included morphology and physiology of proportional body and organ sizes and dimensions, and use of histology to identify stages of gonadal development. Rather than "ecotypes", Clemens et al. described the ocean- and stream-maturing forms as "life histories".

We offer three inter-related reasons to argue that, given the present scientific knowledge regarding the Pacific lamprey, "life histories" is a more appropriate (i.e. conservative) term than "ecotypes" to describe withinspecies variation of maturation schedules:

1. Current information is insufficient to conclude that the ocean- and stream-maturing forms are genetically distinguishable phenotypes occupying different habitats, rather than life histories that display life history trade-offs. Ecotype has been defined as "the product arising as a result of the genotypical response of an ecospecies to a particular habitat" (Turesson 1922, cited in Gregor 1944), and has been used to describe the use of different niches by different forms of the same species (e.g., benthic and limnetic forms of the threespine stickleback Gasterosteus aculeatus ; Baker et al. 2005, and the forms of marine snail Littorina saxatilis that have adapted to the action of waves or predation by crabs; Westram et al. 2014). In lampreys, the term ecotypes has been synonymized with closely-related species pairs, where one species is parasitic as a juvenile, whereas the other species does not have a juvenile feeding stage (Rougemont et al. 2017). Docker and Potter (2019) furthered this argument with the description of two situations where lamprey ecotypes could either be partially or fully reproductively isolated. It is not known whether ocean- and stream-maturing Pacific lamprey occupy different niches at any point in their life cycle, and they co-occur during the freshwater spawning migration. Further, assessment of the neutral alleles of ocean- and stream-maturing Pacific lamprey suggests one panmictic population with no ecotypic differentiation (i.e., no reproductive isolation; Parker et al. 2019). We appreciate that Parker et al. raise the concept of ecotypes when referring to the reproductive variation of ocean- and stream-maturing Pacific lamprey because this has important evolutionary and management implications. However, to conclude that environmental selection was responsible for the evolutionary divergence of ecotypes from a common ancestor would require evidence that these ecotypes encountered some degree of reproductive isolation. Knowledge of the different habitats occupied by each phenotype could greatly enhance monitoring and conservation practices for Pacific lamprey.

2. The names of the phenotypes were chosen by Clemens et al. (2013) to place understanding of them within the template of what is known about other, more well-studied species. The terms ocean- and stream-maturing, and life histories were used by Clemens et al. to place the morphological and physiological information on Pacific lamprey from their study into the context of the extensive evolutionary and ecological literature on Pacific salmon Oncorhynchus spp. (Healey 1991; Hendry and Stearns 2004; Quinn 2005), particularly steelhead O. mykiss (Busby 1996; Brannon et al. 2004; Quinn 2005), as a template to advance understanding of Pacific lamprey. Pacific salmon are sympatric with Pacific lamprey, and steelhead (like Pacific lamprey) spawn in the spring (Quinn 2005; Clemens et al. 2013).

3. "Life history" connotes demographic diversity of phenotypes, which pertains to correlations and tradeoffs among organismal characteristics of mortality, growth, fecundity, age-at-maturation, and body size (Stearns 1989; Winemiller and Rose 1992; Ricklefs and Wikelski 2002). Clemens et al. used the term "life history" to place the within-species diversity they identified for Pacific lamprey into the well-established life history paradigm of Winemiller and Rose (1992). Clemens et al. furthermore used the scheme of Winemiller and Rose to conclude that Pacific lamprey, as a species, is a "periodic" life history strategist.

It will be important to identify the habitat use and extent of distribution of ocean- and stream-maturing life histories of Pacific lamprey. If ocean-maturing lamprey spawn in the same year that they enter freshwater, then one would predict life history differences relative to stream-maturing Pacific lamprey. For example, less time holding in fresh water prior to spawning could yield higher adult survival and fecundity for ocean- 
maturing lamprey relative to stream-maturing lamprey. This is because less time holding in freshwater before spawning could translate to less exposure to predation or other mortality sources, and more energy that could be used for generating a higher fecundity rather than being used to prolong life prior to spawning. We conclude that "ecotype" suggests a fundamental difference in geographic or habitat distribution between ocean- and stream-maturing Pacific lamprey that has not been demonstrated. However, "life histories" more accurately describes the diversity of schedule over which conspecifics mature and reproduce.

\section{Data accessibility statement}

No data are associated with this essay.

\section{Competing interests}

The authors have no competing interests.

\section{Author contributions}

Ben Clemens conceived the idea for this essay and led the writing and editing. Carl Schreck contributed to the ideas, writing, and editing.

\section{Funding information}

Ben Clemens was funded by the Oregon Department of Fish and Wildlife. Carl Schreck was funded by the Department of Fisheries and Wildlife at Oregon State University.

\section{Acknowledgments}

We would like to thank Tom Stahl, Marc Johnson, and David Noakes for providing reviews and comments that improved an early draft of this manuscript.

\section{References}

Arostegui, M. C., T. P. Quinn, L. W. Seeb, J. E. Seeb, G. J. McKinney. 2018. Retention of a chromosomal inversion from an anadromous ancestor provides the genetic basis for alternative freshwater ecotypes in rainbow trout. Molecular Ecology. 28: 1412 - 1427.

Baker, J. A., W. A. Cresko, S. A. Foster, and D. C. Heins. 2005. Life-history differentiation of benthic and limnetic ecotypes in a polytypic population of threespine stickleback (Gasterosteus aculeatus ). Evolutionary Ecology Research. 7: $121-131$.

Baker, J. A., S. A. Foster, D. C. Heins, M. A Bell, and R. W. King. 1998. Variation in female life-history traits among Alaskan populations of the threespine stickleback, Gasterosteus aculeatus L. (Pisces: Gasterosteidae). Biological Journal of the Linnean Society. 63: 141 - 159.

Brannon, E. L., M. S. Powell, T. P. Quinn, and A. Talbot. 2004. Population structure of Columbia River Basin Chinook salmon and steelhead trout. Reviews in Fisheries Science. 12: 99 - 232.

Busby, P. J., T. C. Wainwright, G. J. Bryant, L. J. Lierheimer, R. S. Waples, F. W. Waknitz, and I. V. Lagomarsino. 1996. Status review of west coast steelhead from Washington, Idaho, Oregon, and California. National Oceanographic and Atmospheric Administration Tech. Memo. NMFS-NWFSC-27.

Chavarie, L., K. L. Howland, and W. M. Tonn. 2013. Sympatric polymorphism in Lake Trout: The coexistence of multiple shallow-water morphotypes in Great Bear Lake. Transactions of the American Fisheries Society. 142: $814-823$.

Clemens, B. J., van de Wetering, S., Sower, S. A., and Schreck, C. B. 2013. Maturation characteristics and life-history strategies of the Pacific lamprey, Entosphenus tridentatus . Canadian Journal of Zoology, 91: $775-788$.

Collyer, M. L., M. E. Hall, M. D. Smith, and C. W. Hoagstrom. 2015. Habitat-morphotype associations of Pecos pupfish (Cyprinodon pecosensis ) in isolated habitat complexes. Copeia, 2015(1): 181 - 199. 
Cruz-Font, L., B. J. Shuter, P. J. Blanchfield, C. K. Minns, and M. D. Rennie. 2019. Life at the top: Lake ecotype influences the foraging pattern, metabolic costs and life history of an apex fish predator. Journal of Animal Ecology. 88: $702-716$.

Docker, M. F., J. Hume, and B. J. Clemens. 2015. Introduction: A surfeit of lampreys. Pages 1 - 34 In: M. F. Docker (editor), Lampreys: biology, conservation and control, Volume 1. Springer, Fish and Fisheries Monograph Series, New York.

Docker, M.F., and I. C. Potter. 2019. Life history evolution in lampreys: Alternative migratory and feedings types, in: Docker, M.F. (Ed.), Lampreys: biology, conservation and control. Vol. 2. Fish and Fisheries Series, Vol. 38. Springer, Dordrecht, The Netherlands, pp. $287-410$.

Gregor, J. W. 1944. The ecotype. Biological Reviews. 19: 20 - 30.

Healey, M.C. 1991. Life history of Chinook salmon (Oncorhynchus tshawytscha). In: Groot, C.; Margolis, L., eds. Pacific salmon life histories. Vancouver, BC: University of British Columbia Press: 311-393.

Hendry, A. P., and S.C. Stearns. 2004. Evolution illuminated: salmon and their relatives. Oxford University Press, New York.

Kloh, J. S., C. C. Figueredo, and P. C. Eterovick. 2019. How close is microhabitat and diet association in aquatic ecomorphotypes? A test with tadpoles of syntopic species. Hydrobiologia. 828: $271-285$.

Lessios, H. A., and J. R. Weinberg. 1994. Genetic and morphological divergence among morphotypes of the isopod Excirolana on the two sides of the isthmus of Panama. Evolution. 48: 530 - 548.

Palacios, M. G., A. M. Sparkman, and A. M. Bronikowski. 2012. Corticosterone and pace of life in two life-history ecotypes of the garter snake Thamnophis elegans. General and Comparative Endocrinology. 175: $443-448$.

Parker, K., J. E. Hess, S. R. Narum, and A. P. Kinziger. 2019. Evidence for the genetic basis and epistatic interactions underlying ocean- and river-maturing ecotypes of Pacific Lamprey (Entosphenus tridentatus) returning to the Klamath River, California. Molecular Ecology. 28: 3171 - 3185.

Potter, I. C., H. S. Gill, C. B. Renaud, and D. Haoucher. 2015. The taxonomy, phylogeny, and distribution of lampreys. Pages 35 - 74 In: M. F. Docker (editor), Lampreys: biology, conservation and control, Volume 1. Springer, Fish and Fisheries Monograph Series, New York.

Proćków, M., J. Proćków, P. Błażej, P. Mackiewicz. 2018. The influence of habitat preferences on shell morphology in ecophenotypes of Trochulus hispidus complex. Science of The Total Environment. 630:10361043

Quinn, T. P. 2005. The behavior and ecology of Pacific salmon and trout. University of Washington Press, Seattle.

Ricklefs, R. E., and M. Wikelski. 2002. The physiology/life-history nexus. Trends in Ecology and Evolution. 17: $462-468$.

Rougemont, Q., P.-A. Gagnaire, C. Perrier, C. Genthon, A.-L. Besnard, S. Launey, and G. Evanno. 2017. Inferring the demographic history underlying parallel genomic divergence among pairs of parasitic and nonparasitic lamprey ecotypes. Molecular Ecology. 26: $142-162$.

Schönborn, W., and T. Peschke. 1988. Biometric studies on species, races, ecophenotypes and individual variations of soil-inhabiting Testacea (Protozoa, Rhizopoda), including Trigonopyxis minutansp. and Corythion asperulum nsp. Archiv für Protistenkunde. 136: 345-363.

Segura-Trujillo, C. A., M. R. Willig, and S. T. Álvarez-Castañeda. 2018. Correspondence between ecomorphotype and use of arthropod resources by bats of the genus Myotis . Journal of Mammalogy. 99: 659667. 
Skúlason, S., and T. B. Smith. 1995. Resource polymorphisms in vertebrates. 10: 366 - 370.

Skúlason, S., K. J. Parsons, Richard Svanbäck, K. Räsänen, M. M. Ferguson, C. E. Adams, P.-A. Amundsen, P. Bartels, C. W. Bean, J. W. Boughman, G. Englund, J. Gubrandsson, O. E. Hooker, A. G. Hudson, K. K. Kahilainen, R. Knudsen, B. K. Kristjánsson, C. A-L. Leblanc, Z. Jónsson, G. Öhlund, C. Smith, and S. S. Snorrason. 2019. A way forward with eco evo devo: an extended theory of resource polymorphism with postglacial fishes as model systems. Biological Reviews. 94: 1786 - 1808.

Sorensen, F.E., and D.R. Lindberg. 1991. Preferential predation by American black oystercatchers on transitional ecophenotypes of the limpet Lottia pelta (Rathke). Journal of Experimental Marine Biology and Ecology. 154: 123-136.

Stearns, S. C. 1989. Trade-offs in life-history evolution. Functional Ecology. 3: 259 -268.

Westram, A. M., J. Galindo, M. Alm Rosenblad, J. W. Grahame, M. Panova, and R. K. Butlin. 2014. Do the same genes underlie parallel phenotypic divergence in different Littorina saxatilis populations? Molecular Ecology. 23: $4603-4616$.

Winemiller, K.O., and K. A. Rose. 1992. Patterns of life-history diversification in North American fishes: implications for population regulation. Canadian Journal of Fisheries and Aquatic Sciences. 49: 2196 - 2218.

Wood, C. C., J. W. Bickham, R. J. Nelson, C. J. Foote, and J. C. Patton. 2008. Recurrent evolution of life history ecotypes in sockeye salmon: implications for conservation and future evolution. Evolutionary Applications. 1: $207-221$. 